\title{
Bit wise and delay of vedic multiplier
}

\author{
M Siva Kumar, Sanath kumar Tulasi, N Srinivasulu, Vijaya Lakshmi Bandi, K Hari Kishore
}

Department of ECE, Koneru Lakshmiah Educational Foundation, Vaddeswaram, Guntur,Andhra Pradesh, India 522502

*Corresponding author E-mail: siva4580@kluniversity.in

\begin{abstract}
The Vedic multiplier is derived from the ancient mathematics called Vedic mathematics . The ancient mathematics has different sutras in that we use Urdhva Tiryagbhyam sutra which means clock wise and vertically. As we know that binary multiplication is not possible so that instead we use binary addition or subtraction instead of it. The key process for the multiplication is the speed of the processor. The fastest mode of multiplication is the Vedic multiplier. In this paper we want to show the delay and utilization of components available for the multiplier by executing the code. The comparison of delay from some papers was also proposed in this paper. The research is going on the Vedic mathematics to overcome the problems on the conventional mathematics. In future Vedic multiplier plays an important role in the DSP (Digital Signal Processing).As it is the fastest and efficient mode of operation. In this paper I am calculating the bit wise delay up to 32-bit. The whole analysis was done in Xilinx. The ISM wave forms for every bit up to 32-bit was to be obtained. The utilization, used, available, utilized analysis was also taken. The whole process was done in XILINX software.
\end{abstract}

Keywords: Vedic multiplier, Delay, Digital Signal Processing

\section{Introduction}

Vedic Mathematics is an antiquated arrangement of mathematics ${ }^{3}$ which takes a shot at Vedas which was reproduced by Jagadguru Swami Sri Bharati Krishna Tirthaji Maharaja around 1911 and 1918 from certain Sanskrit scripts. It is perhaps the most refined and profitable numerical structure conceivable One of such effective method has been utilized to plan of a multiplier.

In DSP multipliers are the fundamental components. Increase is the key perspective, whereby change in computational speed of augmentation declines the preparing time of Digital Signal Processors. Multiplier squares makes utilization of quick Fourier changes.. A quicker strategy for increase in light of antiquated Indian Vedic arithmetic is focused on in this paper. Among various strategies of increases in Vedic arithmetic, UrdhvaTiryagbhyam is powerful. UrdhvaTiryagbhyam is a general augmentation recipe pertinent to all instances of multiplication. For expansion of incomplete items in the multiplier[3].

\section{Methodology used}

\subsection{Urdhva tiryagbhyam}

General extension formula utilized is UrdhvaTiryagbhyam Sutra3 proper to all instances of addition. It truly signifies "Vertically and clockwise". How the methodology of the duplication of two, three and four digit numbers is showed up in Fig. 1 using UrdhavaTiryagbhyam Method. The digits on the two closures of the line are duplicated and the outcome is incorporated with the past convey. At whatever point at least three lines are accessible, each one of the outcomes are added to the past convey. The last digit of the number henceforth gained goes about as one of the outcome digit and the rest go about as the convey for the accompanying stride. At first the outcome is taken is zero[3].

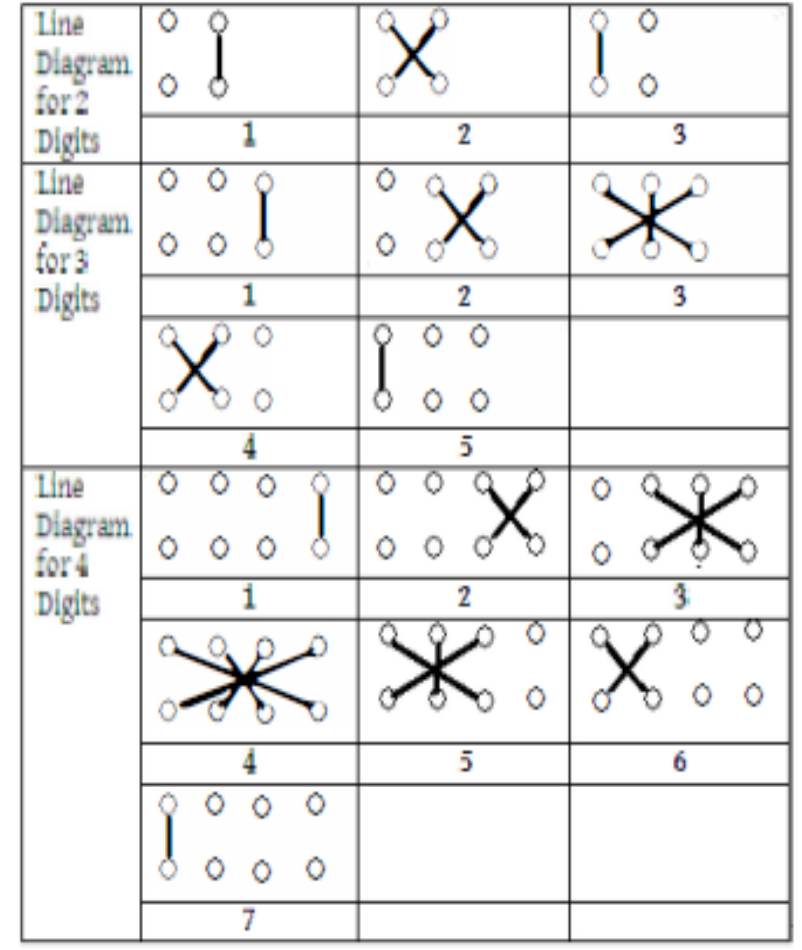

Fig. 1: Vedic multiplier implementation

\subsection{Logisim circuit of $2 * 2$ bit Vedic multiplier}

The last two digits under go AND operation ,after that the second row first digit perform AND operation with first row second digit and the AND operation take place between second row second digit and first row first digit then the summation (half- 
adder)between the two AND operations was performed. At last the first two digits under go AND operation [13].
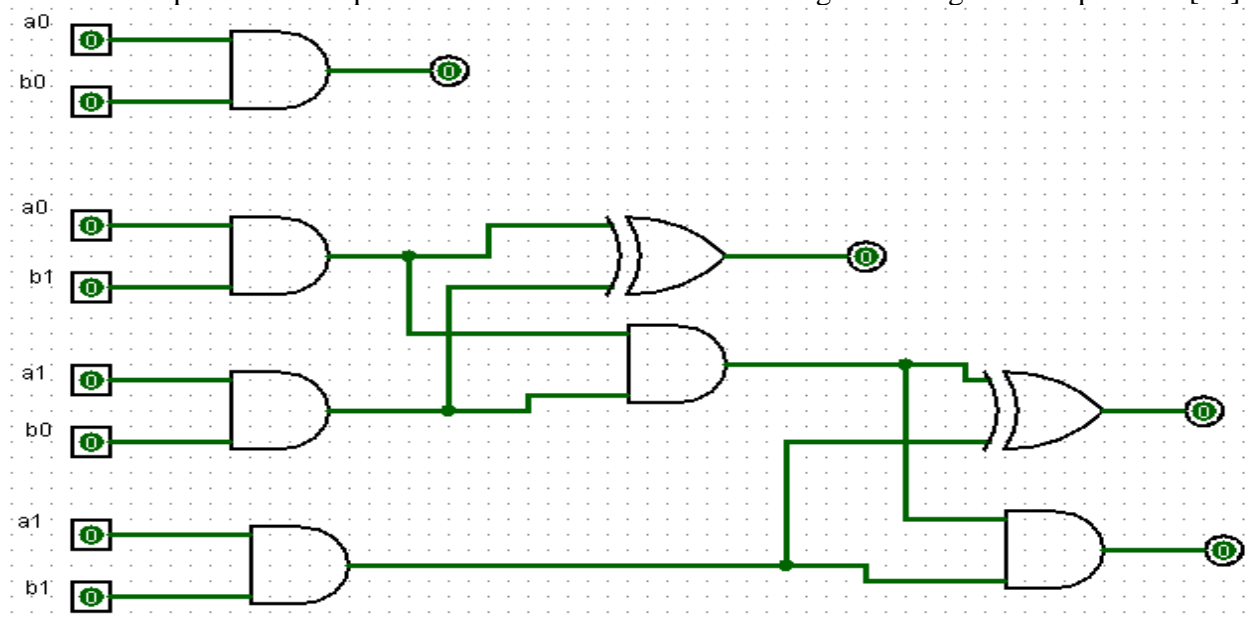

Fig.2.2: Bitimplementation of Vedic multiplier

The whole circuit implemented in logisim was shown in Fig 2. The same process will take place for the 4 -bit, 8-bit, 16-bit, 32-bit. The results are obtained for the individual bits for our requirement.
From the results we are obtaining the delays and utilization of the components was also obtained in the XILINX software while executing ${ }^{6}$.

\section{Xilinx Model Sim Simulations}

\section{$2 * 2$ Vedic multiplier wave forms}
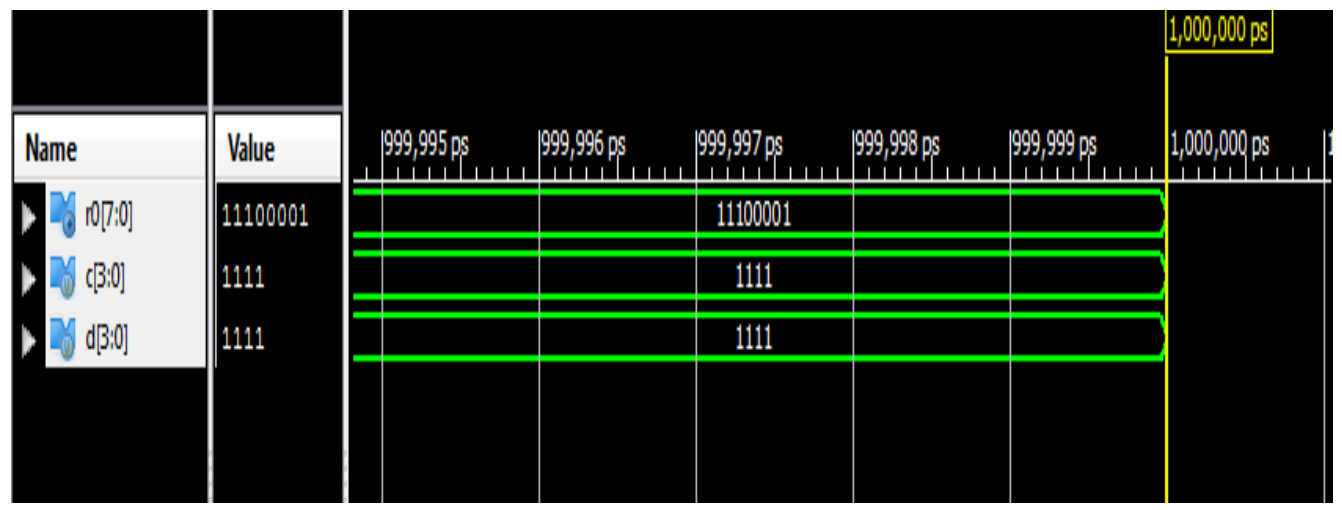

Fig. 3: 2 Bit wave form

Figure 3 describes the movements to build two 2 bit numbers ${ }^{6}$. Changing over the above figure to an equipment comparable we have 3 and gates which will go as 2 bit multipliers and two half adders to add the item to get the last item. Here are the equipment purposes of interest the multiplier Where "an" and "b" are two numbers to be multiply and "q" is the result. With this graph we are presently arranged the code in Verilog effectively utilizing and gates and HA (half adders). To make the design more modular we endeavor code for HA first and a short time later instantiate it to have the last product.

\section{$4 * 4$ vedic multiplier wave forms}
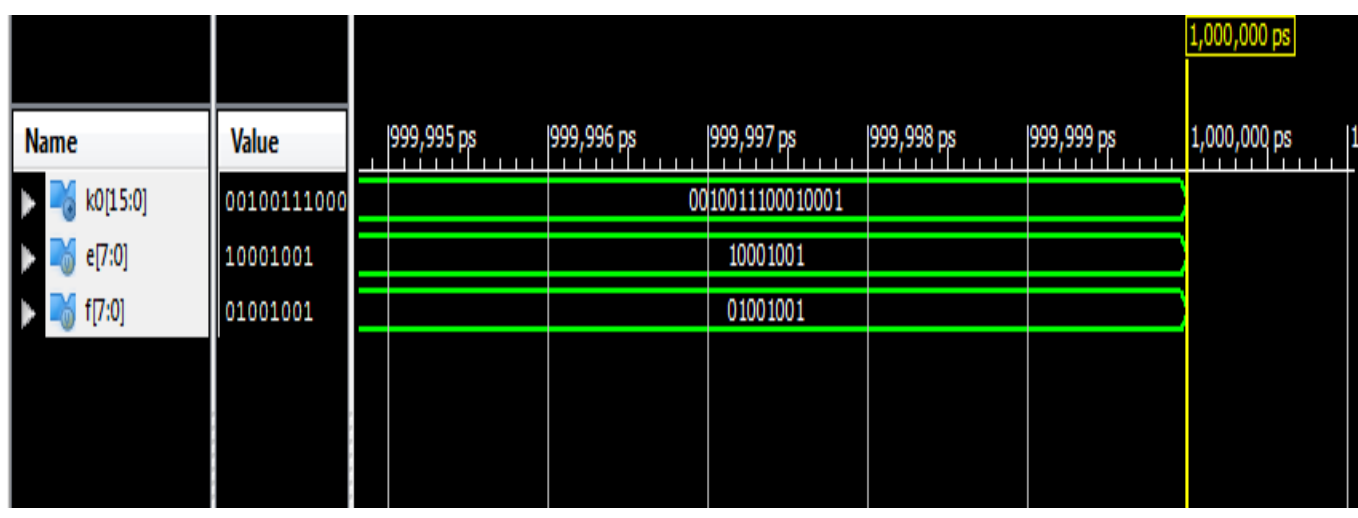

Fig. 4: 4 Bit wave form 
Using 4 such $2 \times 2$ multipliers and 3 adders we can assemble $4 \times 4$ bit multipliers as showed up in the layout. Legitimate instantiating of the $2 \times 2$ multipliers and adders. We have to first make code for 4 bit and 6 bit adders[6].

Each piece as showed up above is $2 \times 2$ bit multiplier. Beginning $2 \times 2$ multiplier inputs are "A1 A0" and "B1 B0". The last square is
$2 \times 2$ piece multiplier with inputs "A3 A2" and "B3 B2". The middle one shows two, $2 \times 2$ bit multiplier with inputs "A3A2" and "B1B0" and "A1A0" and "B3B2". So the last delayed consequence of augmentation, which is of 8 bit, "S7S6S5S4S3S2 S1S0".

\section{$8 * 8$ Vedic multiplier wave form}
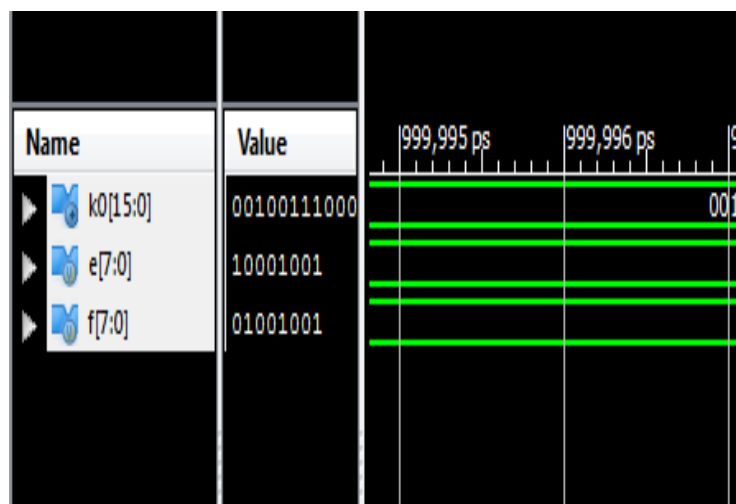

$1,000,000 \mathrm{ps}$

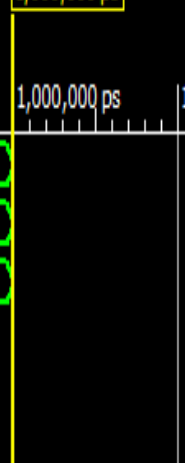

Fig.5: 8 bit wave form

Like the past layout of $4 \times 4$ multiplier, we require 4 such $4 \times 4$ multipliers to make $8 \times 8$ multipliers ${ }^{6}$. Here we need to first arrangement 8 bit and 12 bit adders and by suitable instantiating of the module and associations as showed up in the figure 5 we have made a $8 \times 8$ bit multiplier. As of right now of time it is fundamental for you to try and confirm the RTL code and check if the equipment is according to our design. Arrangement Ahead apparatus by Xilinx gives better perspective of the hardware output with configuration elaborate alternative. Elude the development tree chart to know the methodology for $8 \times 8$ multiplier.

\section{$16 * 16$ Vedic multiplier wave form}
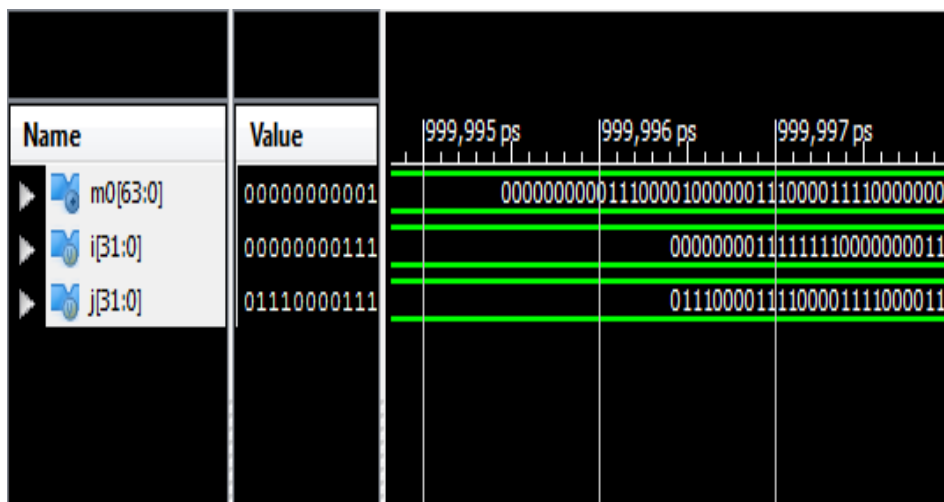

Fig. 6: 16-bit wave form.

We take after the same stratage as though there ought to be an event of past multipliers and make $16 \times 16$ multipliers appeared in fig 6 . The framework of $16 \times 16$ piece is a practically identical action of $8 \times 8$ squares in an advanced path as the introductory stage in the setup of $16 \times 16$ square will be assembling the 8 bit (byte) of each 16 bit information. These lower and upper bytes sets of two

inputs will shape vertical and transversely thing terms. Each information byte is care with by an alternate $8 \times 8$ Vedic 15 multiplier to convey sixteen halfway thing pushes. These partial things columns are then incorporated a 16-bit carry look ahead adder in ideally to make last bits ${ }^{6}$

\section{$32 * 32$ Vedic multiplier waveform}




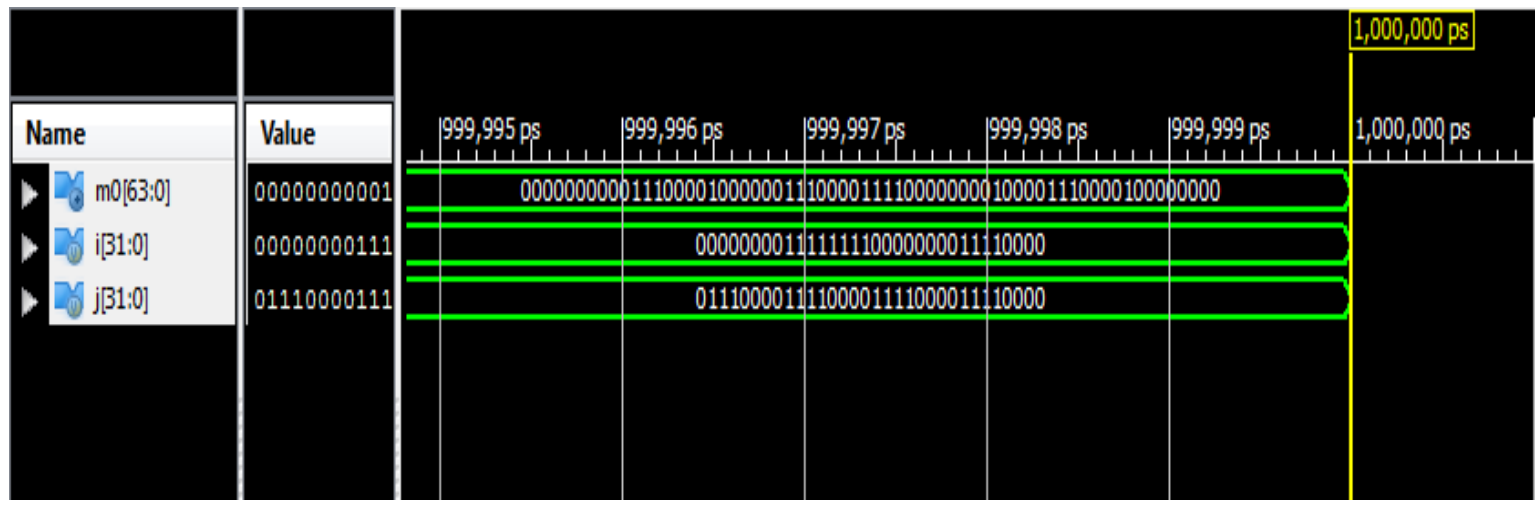

Fig. 7: 32-bit wave form.

Same like the above we can obtain this wave forms from the $16 * 16$ Vedic multiplier as shown in fig7.By using the format of the Vedic multiplier we can obtain the wave forms for the higher order also [6].

\section{RTL Schematics of vedic multiplier}

The RTL schematic for the Vedic multiplier was inter related to each other individually the 2-bit was used to obtain 4-bit,the 4-bit is used to obtain8-bit,the 8-bit is used to obtain 16-bit,16-bit is used to obtain32-bit. Without the formation of one bit another bit was not obtained. The RTL schematic of 2-bit was stated below figure8.

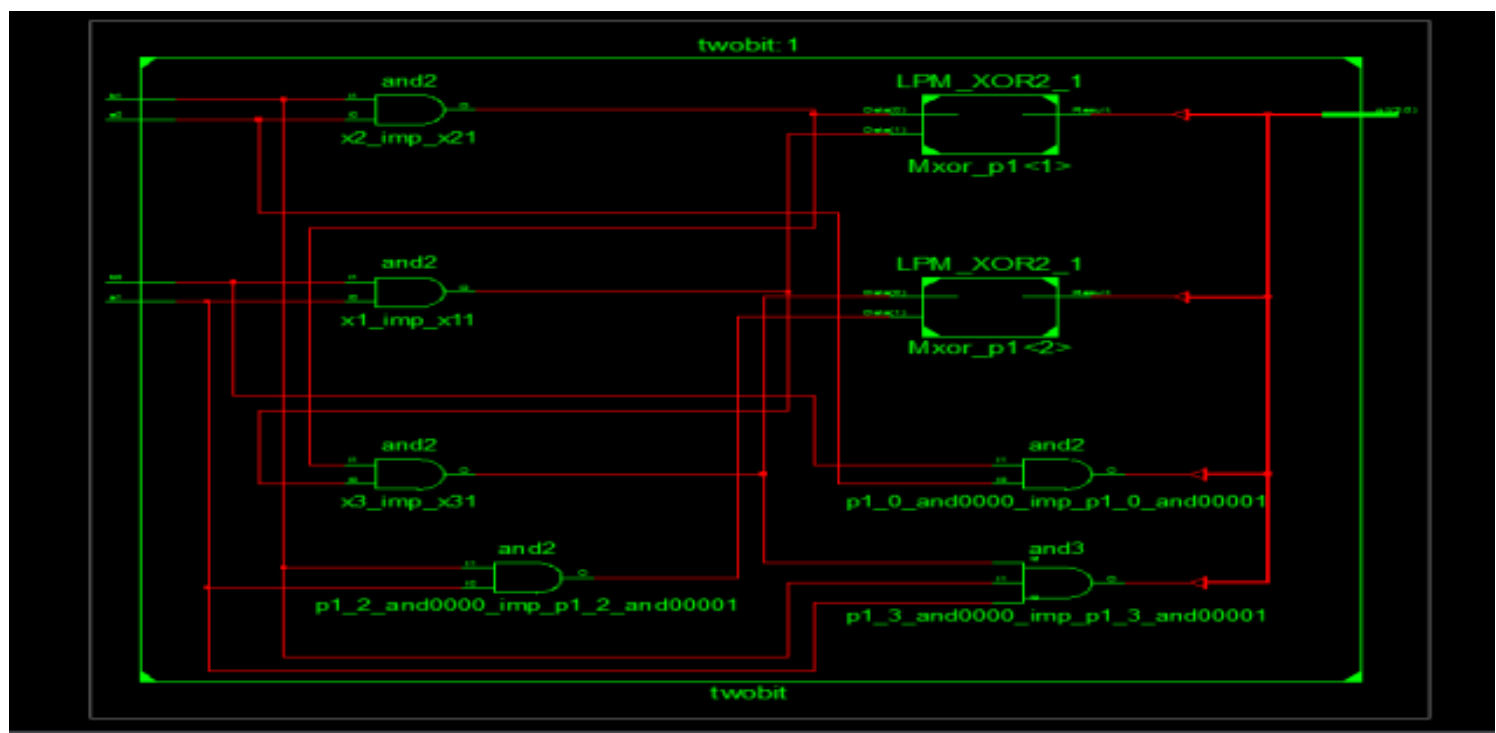

Fig. 8. RTL schematic of 2-bit vedic multiplier.

\section{Delay analysis with respect to different papers and different multipliers}

\section{2*2 Multiplier:}

The delay of 2-bit Vedic multiplier was shown below Maximum combinational path delay:6.376ns

\section{4*4 Multiplier:}

The delay of 4-bit Vedic multiplier was shown below

Maximum combinational path delay: $12.542 \mathrm{~ns}$

8*8 Multiplier:

The delay of 8-bit Vedic multiplier was shown below Maximum combinational path delay:19.416ns

\section{6*16 Multiplier:}

The delay of 16-bit Vedic multiplier was shown below Maximum combinational path delay: $25.825 \mathrm{~ns}$

\section{2*32 multiplier:}

The delay of 32-bit Vedic multiplier was shown below Maximum combinational path delay:32.237ns
Table 1: Table showing delay comparison for 16 bit and 8 bit in previous papers

\begin{tabular}{|l|l|}
\hline Bit wise & Delay \\
\hline 16-bit & $50.952 \mathrm{~ns}$ \\
\hline 8 -bit & $23.64 \mathrm{~ns}$ \\
\hline
\end{tabular}

Table 2: Table showing delay comparison for 16 bit and 8 bit in our present papers

\begin{tabular}{|l|l|}
\hline Bit wise & Delay \\
\hline $16-$ bit & $25.825 \mathrm{~ns}$ \\
\hline 8 -bit & $19.416 \mathrm{~ns}$ \\
\hline
\end{tabular}

For 16-bit Vedic multiplier using urdhavaTiryagbhyam multiplier in ${ }^{6}$ was $50.952 \mathrm{~ns}$ but in our model we get the delay of $25.825 \mathrm{~ns}$.

For 8-bit Vedic multiplier using urdhvaTiryagbhyam multiplier in ${ }^{6}$ was $23.644 \mathrm{~ns}$ but in our model we get the delay of $19.416 \mathrm{~ns}$.

We can say that utilization of the components and delay is less in the proposed paper than the reference paper. 


\section{Advantage}

The speed increases for the reversible logic of the multiplication process will occur in same time so by this system reaction is not different for different inputs and all will gives output at same time.

\section{Limitation}

In this the limitation is that if we increase the number of bits that appended by this the number of flip-flops increases and by this the size increases by this the Power consumption increases.

\section{Applications}

- Micro processors

- Filtering process (FIR)

- Digital signal processors

- IF stages of the receiver

\section{Conclusion and future scope}

The conventional multiplication methodology requires additional time and territory on silicon than Vedic calculations. All the more fundamentally dealing with speed increments with the bit length This will help at long last to speed the preparing work, as it some assistance with being certainly appreciated that the multiplier is the real building square of FFT.

Multipliers can be designed to take after the strategy for Vedic multiplication. The disadvantage of designing any higher bit multiplier is that the lower bit multipliers can't be cascaded to acquire a multiplier capable for increasing more number of bits, as an account of adders. The Vedic multiplier can be utilized to solve this issue. For instance we can outline 64-bit Binary multiplier can be utilized to design a 32-bit binary multiplier.

\section{References}

[1] Budda Nagendra Reddy and P. Augusta SophyBeulet, An Efficien Multi-Precision Floating Point Adder and Multiplier, Indian Journal of Science and Technology, October 2015.

[2] S. Subathradevi1 and C. Vennila ,Modified Architecture for Binary Array Multiplier with Reduced Delay using Tristate Buffers,Indian Journal of Science and Technology,september 2015.

[3] PrakashNarchiSiddalingesh $S$ kerurJayashree $C$ NidagundiHarish $M$ Kittur and Girish V A,:Implementation of Vedic Multiplier for Digital Signal processing.IJCA proceedings on International Conference on VLSI, CommunicationsandInstrumentation",2015

[4] Dr. K.S. Gurumurthy, M.S Prahalad "Fast and Power Efficien $16 \times 16$ Array of Array Multiplier using Vedic Multiplication.

[5] M. Ramalatha, K. Deena Dayalan, P. Dharani, S. Deborah Priya," High Speed Energy Efficient ALU Design using Vedic5.Multiplication Techniques ", ACTEA 2009.

[6] http://verilogcode.blogspot.in/2014/01/design-and-implementationof-16-bit.html

[7] R. Sakthivel, M. Vanitha and Sneha Singh ,Low Leakage Power Vedic Multiplier using Standard Cell Design Indian Journal of Science and Technology, Sep 2015

[8] Poornima M, Shivaraj Kumar Patil, Shivukumar, Shridhar K P Sanjay H," Implementation of Multiplier using Vedic Algorithm"IJITEE 2013.

[9] C.Sheshavali, K.Niranjankumar "Design and Implementation of Vedic Multiplier"IJERD 2013.

[10] PushpalataVerma" Design of $4 \times 4$ bit Vedic Multiplier using EDA Tool" IJCA 2012

[11] Manoranjanpradhan,Rutuparnapanda,sushantakumarsahu," Speed Comparison of 16x16 Vedic Multipliers", International Journal of Computer Applications, Volume 21- No.6, May 2011

12] Ch.Harish Kumar, "Implementation and analysis of power, area and delay of array, urdhvanikhilamvedic multipliers", International Journal of Scientific and Research Publications, Volume 3, Issue 1, January 2013
[13] Badal Sharma," Design and Hardware Implementation Of 128-bit Vedic Multiplier", International journal for advance research in engineering and technology, Volume 1, June 2013.

[14] Dr. Seetaiah Kilaru, Hari Kishore K, Sravani T, Anvesh Chowdary L, Balaji T "Review and Analysis of Promising Technologies with Respect to fifth Generation Networks", 2014 First International Conference on Networks \& Soft Computing, ISSN:978-1-47993486-7/14,pp.270-273,August2014

[15] Meka Bharadwaj, Hari Kishore "Enhanced Launch-Off-Capture Testing Using BIST Designs" Journal of Engineering and Applied Sciences, ISSN No: 1816-949X, Vol No.12, Issue No.3, page: 636643, April 2017.

[16] N Bala Dastagiri, Kakarla Hari Kishore "Reduction of Kickback Noise in Latched Comparators for Cardiac IMDs" Indian Journal of Science and Technology, ISSN No: 0974-6846, Vol No.9, Issue No.43, Page: 1-6, November 2016.

[17] A.Murali, K Hari Kishore, D Venkat Reddy "Integrating FPGAs with Trigger Circuitry Core System Insertions for Observability in Debugging Process" Journal of Engineering and Applied Sciences, ISSN No: 1816-949X, Vol No.11, Issue No.12, page: 2643-2650, December 2016.

[18] Mahesh Mudavath, K Hari Kishore "Design of CMOS RF FrontEnd of Low Noise Amplifier for LTE System Applications Integrating FPGAs" Asian Journal of Information Technology, ISSN No: 1682-3915, Vol No.15, Issue No.20, page: 4040-4047, December 2016

[19] P Bala Gopal, K Hari Kishore, B.Praveen Kittu "An FPGA Implementation of On Chip UART Testing with BIST Techniques", International Journal of Applied Engineering Research, ISSN 0973 4562, Volume 10, Number 14 , pp. 34047-34051, August 2015.

[20] S Nazeer Hussain, K Hari Kishore "Computational Optimization of Placement and Routing using Genetic Algorithm" Indian Journal of Science and Technology, ISSN No: 0974-6846, Vol No.9, Issue No.47, page: 1-4, December 2016.

[21] N Bala Gopal, K Hari Kishore "Analysis of Low Power Low Kickback Noise in Dynamic Comparators in Pacemakers" Indian Journal of Science and Technology, ISSN No: 0974-6846, Vol No.9, Issue No.44, page: 1-4, November 2016.

[22] Rajesh, M., and J. M. Gnanasekar. \&quot;Congestion control in heterogeneous wireless ad hoc network using FRCC.\&quot; Australian Journal of Basic and Applied Sciences 9.7 (2015): 698 702.

[23] S.V.Manikanthan and V.Rama"Optimal Performance Of Key Predistribution Protocol In Wireless Sensor Networks" International Innovative Research Journal of Engineering and Technology ,ISSN NO: 2456-1983,Vol-2,Issue -Special -March 2017

[24] T. Padmapriya and V. Saminadan, "Inter-cell Load Balancing Technique for Multi- class Traffic in MIMO - LTE - A Networks", International Conference on Advanced Computer Science and Information Technology, Singapore, vol.3, no.8, July 2015. 